\title{
Diversity and enterotype in gut bacterial community of adults in Taiwan
}

\author{
Chao Liang ${ }^{1+}$, Han-Chi Tseng ${ }^{2 \dagger}$, Hui-Mei Chen ${ }^{1+}$, Wei-Chi Wang ${ }^{3}$, Chih-Min Chiu ${ }^{3}$, Jen-Yun Chang ${ }^{3}$, Kuan-Yi Lu ${ }^{3}$, \\ Shun-Long Weng ${ }^{5,6,7}$, Tzu-Hao Chang $^{8}$, Chao-Hsiang Chang ${ }^{9}$, Chen-Tsung Weng ${ }^{3}$, Hwei-Ming Wang ${ }^{3}$ \\ and Hsien-Da Huang ${ }^{1,4,10^{*}}$
}

From The 27th International Conference on Genome Informatics

Shanghai, China. 3-5 October 2016

\begin{abstract}
Background: Gastrointestinal microbiota, particularly gut microbiota, is associated with human health. The biodiversity of gut microbiota is affected by ethnicities and environmental factors such as dietary habits or medicine intake, and three enterotypes of the human gut microbiome were announced in 2011. These enterotypes are not significantly correlated with gender, age, or body weight but are influenced by long-term dietary habits. However, to date, only two enterotypes (predominantly consisting of Bacteroides and Prevotella) have shown these characteristics in previous research; the third enterotype remains ambiguous. Understanding the enterotypes can improve the knowledge of the relationship between microbiota and human health.
\end{abstract}

Results: We obtained 181 human fecal samples from adults in Taiwan. Microbiota compositions were analyzed using next-generation sequencing (NGS) technology, which is a culture-independent method of constructing microbial community profiles by sequencing 165 ribosomal DNA (rDNA). In these samples, 17,675,898 sequencing reads were sequenced, and on average, 215 operational taxonomic units (OTUs) were identified for each sample. In this study, the major bacteria in the enterotypes identified from the fecal samples were Bacteroides, Prevotella, and Enterobacteriaceae, and their correlation with dietary habits was confirmed. A microbial interaction network in the gut was observed on the basis of the amount of short-chain fatty acids, pH value of the intestine, and composition of the bacterial community (enterotypes). Finally, a decision tree was derived to provide a predictive model for the three enterotypes. The accuracies of this model in training and independent testing sets were 97.2 and 84.0\%, respectively.

Conclusions: We used NGS technology to characterize the microbiota and constructed a predictive model. The most significant finding was that Enterobacteriaceae, the predominant subtype, could be a new subtype of enterotypes in the Asian population.

Keywords: Enterotype, 165 rDNA, Next-generation sequencing, Gut microbiome, Predictive model

\footnotetext{
* Correspondence: bryan@mail.nctu.edu.tw

${ }^{\dagger}$ Equal contributors

'Institute of Bioinformatics and Systems Biology, National Chiao Tung

University, HsinChu, Taiwan

${ }^{4}$ Department of Biological Science and Technology, National Chiao Tung

University, HsinChu, Taiwan

Full list of author information is available at the end of the article
} 


\section{Background}

Microorganisms inhabit various sites of the human body [1]. The largest number of microorganisms is found in the gut [1]. The gut microbiome is associated with human health [2]. For example, the gastrointestinal microbiome affects human physiological functions such as immune function and inflammation suppression, food decomposition and nutrient absorption, regulation of blood substrate via the nervous and/or endocrine system, and recovery rate from bacterial infection [3]. However, some of the underlying mechanisms remain unclear. Enterotypes of the human gut microbiome are not associated with gender, age, or body weight but are influenced by long-term dietary habits. Therefore, we aimed to identify the enterotypes of adults in Taiwan by next-generation sequencing (NGS).

Since the Human Microbiome Project (HMP) was launched by the National Institutes of Health in 2008, NGS has been widely used to study the human microbiome [4]. One of the benefits of NGS is that it is a cultureindependent method that can be used to characterize microbial community profiles by sequencing of $16 \mathrm{~S}$ ribosomal DNA (rDNA). In addition, hundreds to thousands of bacteria can be identified at a time on sequencing $16 \mathrm{~S}$ rDNA by NGS. Thus, variations in bacteria among different samples can be determined by comparing their quantitative profiles [5].

In 2011, three enterotypes of the human gut microbiome were identified from 261 human fecal samples from European individuals. The major bacteria in these enterotypes were Bacteroides, Prevotella, and Ruminococcus [6]. This finding was subsequently validated by another approach using the same HMP dataset [7]. However, after identifying the long-term dietary habits in subjects, another study only observed Bacteroides and Prevotella enterotypes in their dataset and reported that Ruminococcus was an ambiguous enterotype [8]. The HMP dataset included gut microbiota that was rich in saturated fats and animal protein, whereas the latter study included microbiota from individuals with plant-based diets that were low in meat and high in carbohydrates [9]. In 2012, two other groups also reported that Ruminococcus could not be clearly classified in their datasets, and Firmicutes were identified as the dominant species in those studies [10, 11].

Based on the data from these previous studies, we were interested in determining if Ruminococcus is an enterotype in the gut microbiota of Taiwanese individuals. To this end, 181 human fecal samples from adults in Taiwan were collected, and the V4 regions of the $16 \mathrm{~S}$ rDNA gene were sequenced through paired 150-cycle reads using the Illumina MiSeq system. A total of $17,675,898$ sequencing reads were sequenced in 181 samples, and 215 operational taxonomic units (OTUs) were identified in each sample on average. The most abundant bacteria identified in the fecal samples were Bacteroides, Prevotella, and Enterobacteriaceae, and their correlation with dietary habits was confirmed. A decision tree model of these three enterotypes was constructed. The accuracies of this model in training and independent testing sets were 97.2 and $84.0 \%$, respectively.

The most significant finding in our study was the identification of Enterobacteriaceae as one of the predominant subtypes in the gut microbiota. This species may be a new subtype of enterotypes in the Asian population.

\section{Results and discussion}

Sequencing data statistics

We conducted $17,675,898$ sequencing reads on 181 stool samples. After filtering the sequences that did not fit the criteria, we further analyzed 16,474,959 sequencing reads. After taxonomy assignment, 9,133,183 sequencing reads were aligned to genes in the $16 \mathrm{~S}$ rDNA database that had a sequence similarity of at least 97\%; 215 OTUs for each sample were identified on average. Detailed information on the sequencing reads is listed in Additional file 1: Table S1.

\section{Enterotype identification in the fecal samples}

Nine $\beta$-diversity matrices were used to identify the enterotypes in fecal samples via three clustering methods: hierarchical clustering $(\mathrm{HC})$, partitioning around medoids (PAM), and k-means (Table 1). The optimal number of clusters was two using unweighted UniFrac distance. Principal coordinate analysis ( $\mathrm{PCoA})$ was also used to observe patterns in the stool samples. According to the Euclidean distance matrix, two clusters were shown in opposite areas (Fig. 1a, green and red dots), the major bacteria in which were Bacteroides and Prevotella. In contrast, the other samples (black dots in Fig. 1a) were scattered. Compared

Table 1 Summary of optimal cluster numbers

\begin{tabular}{lllll}
\hline & $\mathrm{HC}^{\mathrm{ac}}$ & $\mathrm{PAM}^{\mathrm{a}}$ & $\mathrm{PAM}^{\mathrm{b}}$ & Kmeans $^{\mathrm{b}}$ \\
\hline Weighted UniFrac & $3(0.339)$ & $3(0.350)$ & $3(0.353)$ & $3(0.354)$ \\
Altgower & $2(0.280)$ & $2(0.161)$ & $2(0.305)$ & $2(0.309)$ \\
Bray & $2(0.302)$ & $2(0.297)$ & $2(0.309)$ & $2(0.309)$ \\
Jaccard & $2(0.221)$ & $2(0.216)$ & $2(0.225)$ & $2(0.225)$ \\
Kulczynski & $2(0.302)$ & $2(0.297)$ & $2(0.309)$ & $2(0.309)$ \\
Maximum & $2(0.459)$ & $2(0.451)$ & $2(0.464)$ & $2(0.468)$ \\
Pearson & $8(0.571)$ & $2(0.614)$ & $2(0.622)$ & $2(0.622)$ \\
Horn & $2(0.494)$ & $2(0.596)$ & $2(0.600)$ & $2(0.600)$ \\
Euclidean & $2(0.267)$ & $2(0.418)$ & $2(0.419)$ & $2(0.418)$ \\
\hline
\end{tabular}

The first number of each cell is the optimal cluster number and the second number is the Silhouette score. The optimal cluster number corresponding to the maximum score was picked from a limited series of cluster numbers $(\mathrm{k} \leq 10)$

${ }^{\mathrm{a}}$ Beta matrix was applied as the input

${ }^{b}$ Coordinate (PC1-PC3) of each sample was applied as the input. Coordinate was generated by the classical multidimensional scaling method from the beta matrix

${ }^{\mathrm{c}} \mathrm{HC}$, Hierarchical clustering 

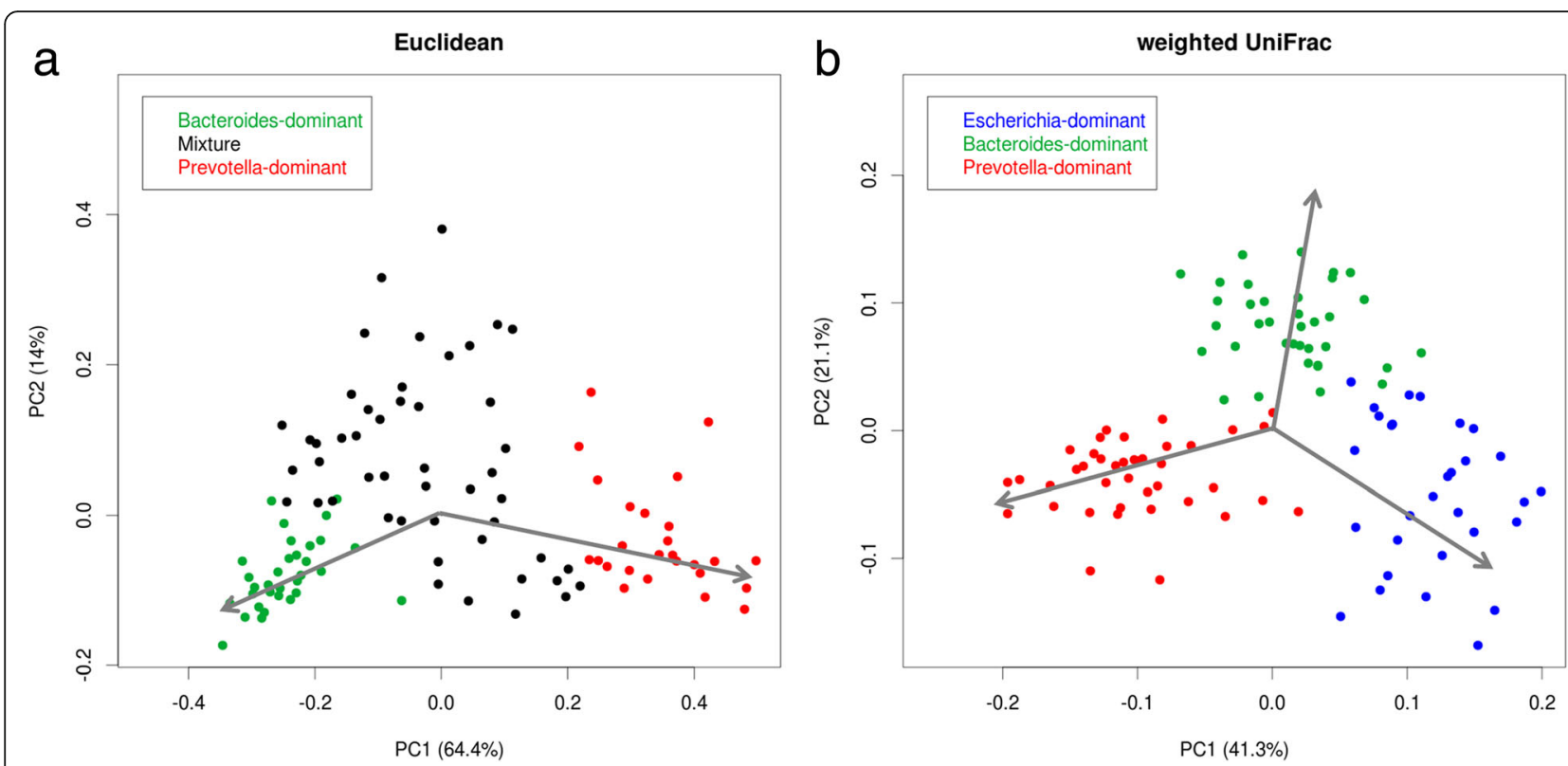

Fig. 1 PCoA results based on different algorithms: a Euclidean and $\mathbf{b}$ Weighted UniFrac

with the PCoA results of weighted UniFrac distance (Fig. 1b), the samples were grouped into three clusters, and the predominant bacterium in the third cluster was identified as Escherichia. Figure 2 shows the results of $\mathrm{HC}$ of the stool samples. The unsupervised classification method produced a dendrogram of the clustering results (Fig. 2a), and the results of four clustering algorithms of weighted UniFrac showed high concordance (Fig. 2b). Then three enterotypes were classified as containing Escherichia (enterotype 1), Bacteroides (enterotype 2), and Prevotella (enterotype 3) (Fig. 2c), and two small unconventional regions were found. One region was located in the Prevotella-predominant group ("star region" in Fig. 2b) and contained six samples. The abundance of Prevotella and Bacteroides showed a high positive correlation within the samples. In addition, nine samples, with a relatively high abundance of Bacteroides and low abundance of Escherichia, were classified into the Bacteroides-predominant group using the PAM method ("triangle region" in Fig. 2b). Moreover, the abundance of bacteria at the family level was similar to that at the genus level (Fig. 2d). Hence, the results of clustering may be improved on the basis of the family level.

\section{Characteristics of the enterotypes}

The bar charts (Fig. 3a) illustrate the relative abundance of bacteria among the three enterotypes. In enterotypes 1 and 3, the major bacteria were Bacteroides, Escherichia, and Prevotella, which accounted for over half of the bacteria. In contrast, in enterotype 2, Prevotella were not the most abundant bacteria; the most abundant bacteria in this enterotype were Bacteroides, which accounted for almost half of the bacteria. In enterotypes 2, the abundance of Bacteroides was at least twofold higher than that of Prevotella. The relative abundance of Escherichia and Bacteroides in enterotype 1 was almost equal. Figure $3 \mathrm{~b}$ shows the predominance of the three most abundant bacteria in the three enterotypes. The abundance of Escherichia was almost similar in both enterotypes 2 and 3, the abundance of Bacteroides in enterotype 1 was higher than that in enterotype 3 , and the abundance of Prevotella in enterotype 1 was higher than that in enterotype 2. The proportion of Bacteroides was inversely correlated with that of Prevotella $(p<$ $0.001, \mathrm{R}=-0.85$ ) (Fig. 4). These results correspond to the bacterial abundance in enterotypes 2 and 3 .

The Shannon diversity index and composition of facultative, anaerobic, and aerobic bacteria in the three enterotypes are shown in Figs. 5 and 6, respectively. The Escherichia-predominant enterotype (enterotype 1) had a higher Shannon index than the other enterotypes $(p<0.001)$. Facultative and anaerobic bacteria showed an overwhelming majority (abundance of $98.3 \%$ on average) with a strong negative correlation $(p<0.0001, \quad \mathrm{R}=$ $-0.94)$. It has been shown that anaerobic bacteria are the predominant bacteria at the endpoint of the gastrointestinal tract [12]. In our study, loss of anaerobicity was observed in the Escherichia-predominant enterotype, in which the abundance of facultative bacteria was significantly higher than that of the other enterotypes $(p<$ 0.0001 , fold change $>2.5$ ) and significantly corresponded with a lower number of anaerobic bacteria $(p<0.0001)$. 

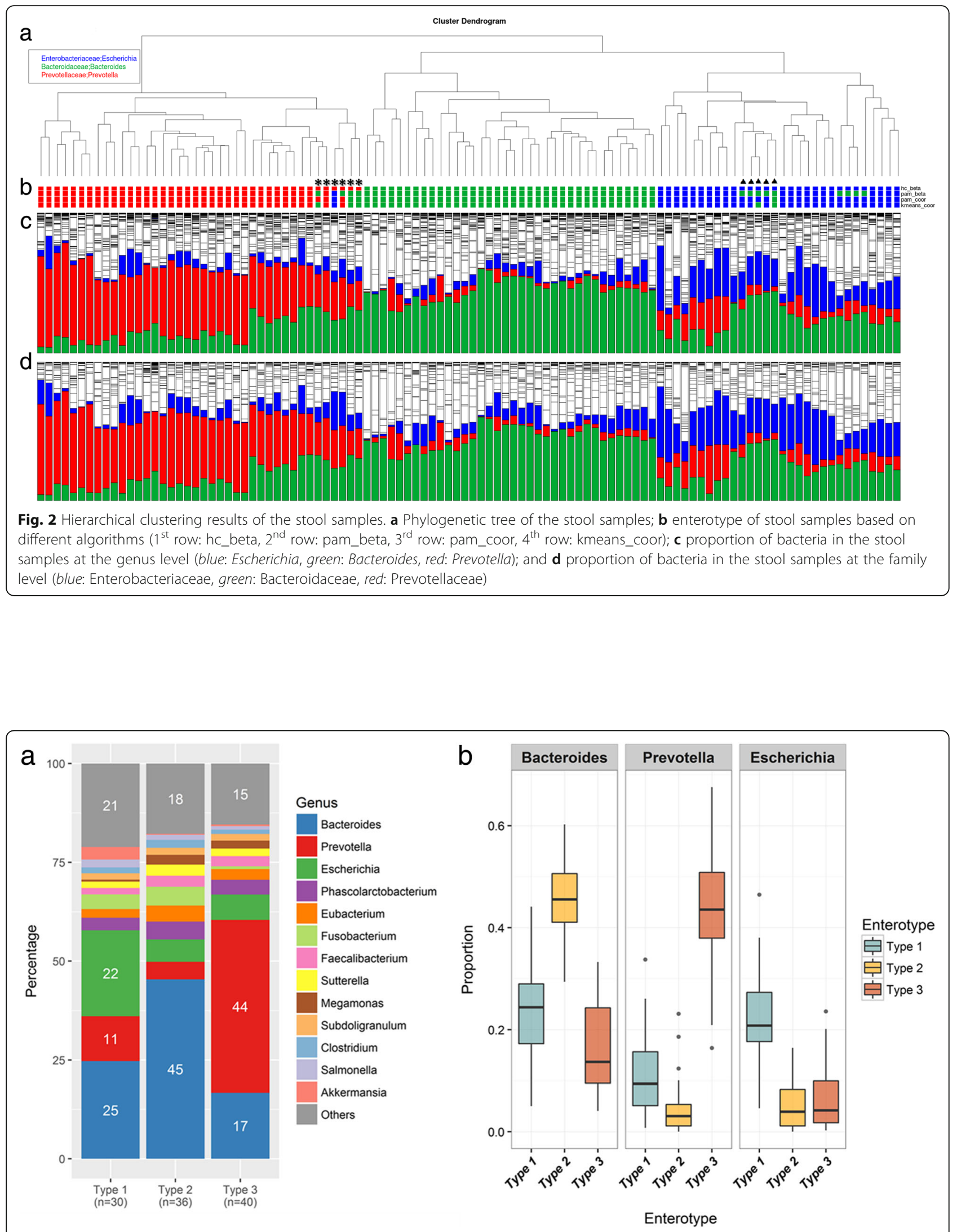

Fig. 3 Bacterial community of three enterotypes: a bacterial proportion in the three enterotypes and $\mathbf{b}$ major bacteria in the three enterotypes 


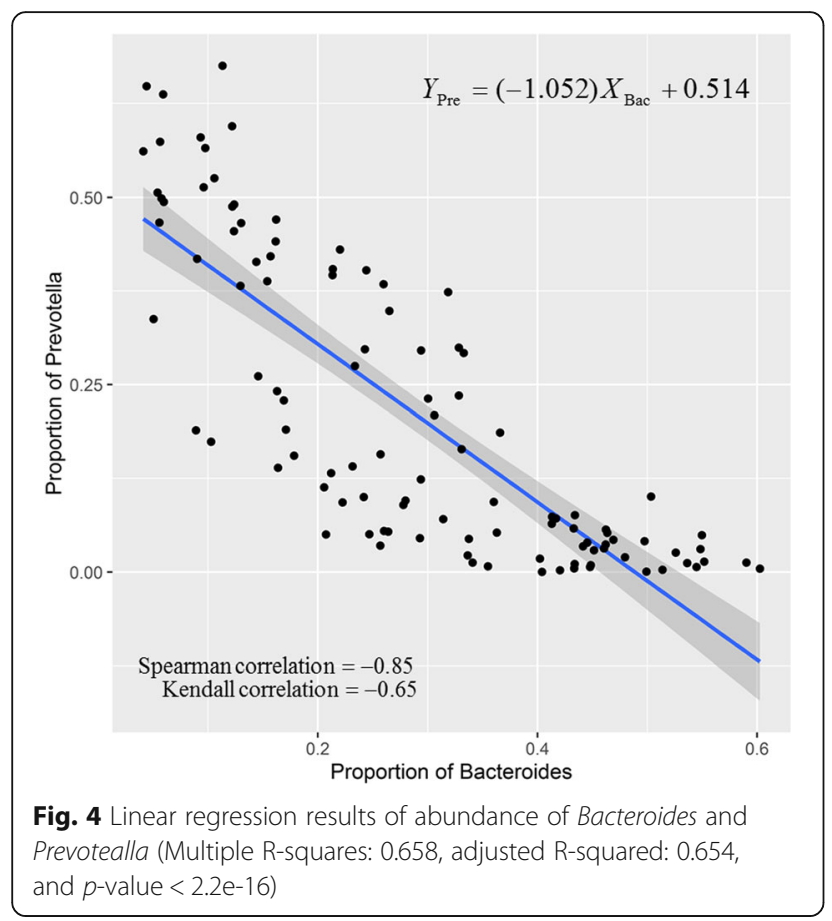

The facultative/anaerobic ratio (defined as the F/A ratio) was also significantly larger in enterotype 1 than in the other two enterotypes $(p<0.0001$, fold change $=3.85)$.

\section{Enterotype phenotypes}

The questionnaire given to the study subjects included questions about three major determinants regarding the samples. The first was the shape of feces, which was categorized by participants according to the Bristol stool scale. Scores of 1-3 represented "hard" stool, a score of four represented "mid," and scores of 5-7 represented "watery" that had a high water content [13]. The second determinant was the frequency of excretion. At least one excretion every 2 days was designated "D1+," excretion two to three times a week was designated "D05," and excretion once a week or less referred to as "constipation." The third variable was "protein type," which referred to the major source of protein in daily diets: the non-red meat group included individuals who eat beans/vegetables, fish, and poultry and the red meat group included individuals who mostly eat livestock.

Table 2 demonstrates the association between enterotypes and several other factors such as gender, protein, shape, and stool frequency. Twice as many females as
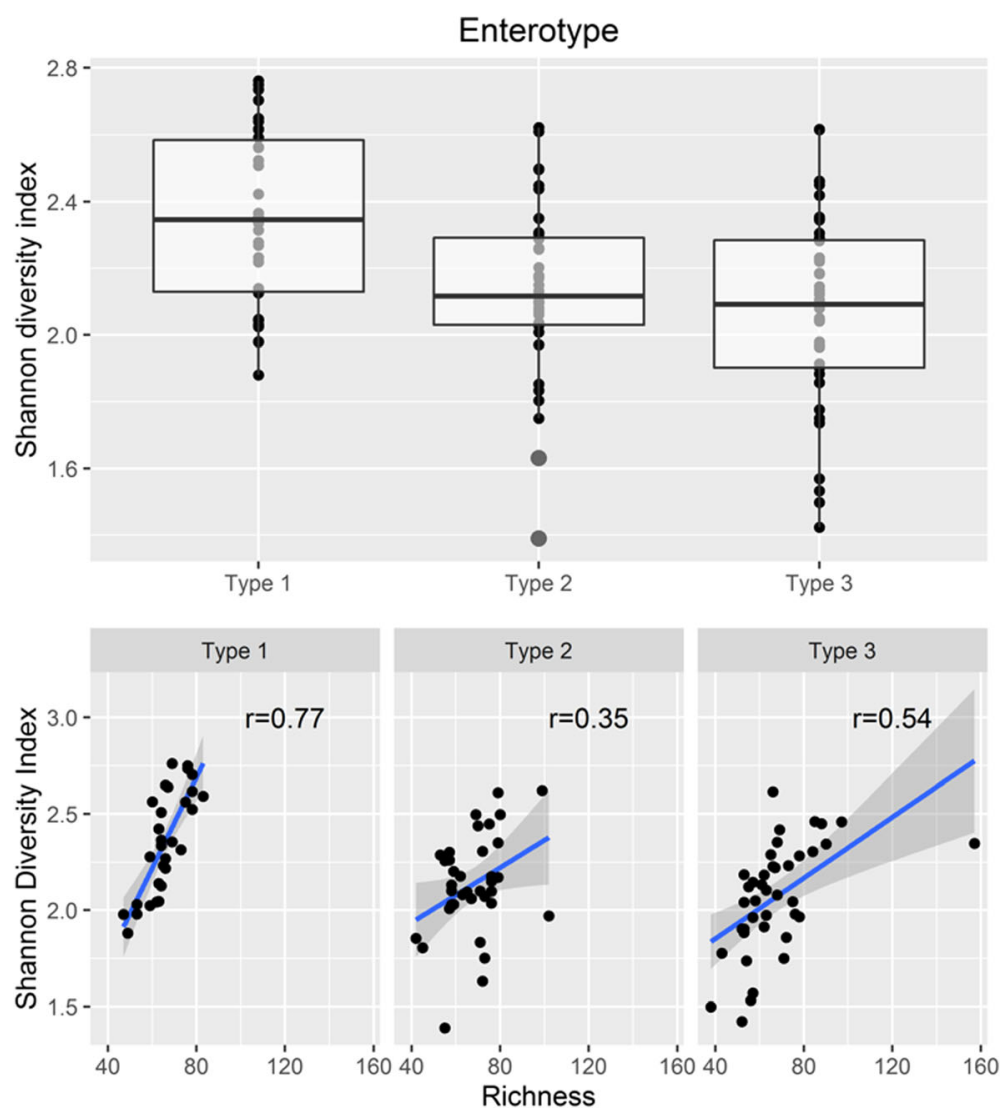

Fig. 5 Distribution of the Shannon diversity index of three enterotypes and the correlation of richness and Shannon diversity index 


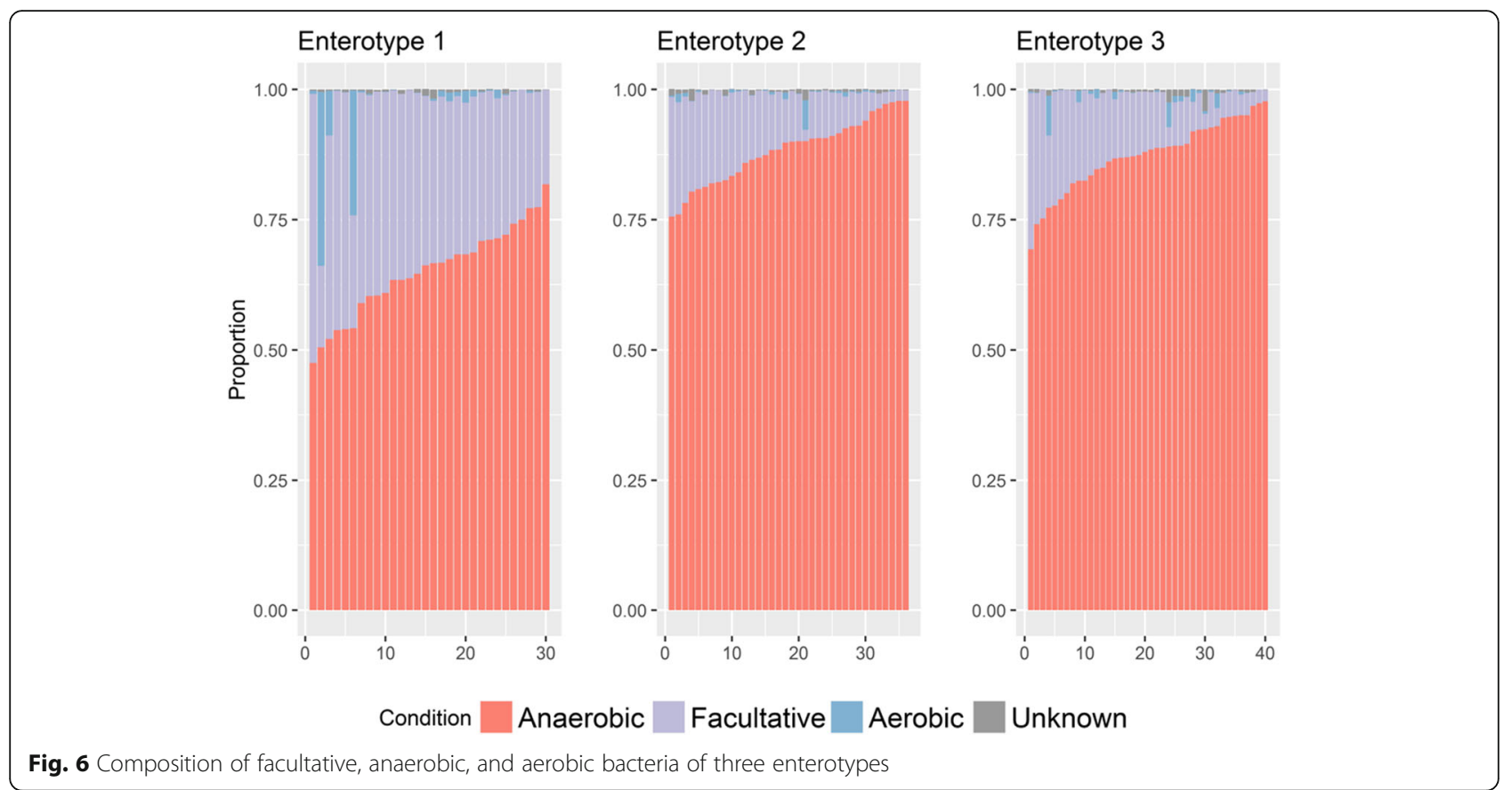

males were classified as enterotype 2 ( $\mathrm{T} 2$ vs. $\mathrm{T} 1+\mathrm{T} 3$, $p=0.02$ ). In both enterotypes 1 and 3 , the gender ratio was close to 1:1 (Table 2. Additional file 2: Figure S1a), which is similar to the results of previous studies $[7,10]$. The number of individuals who consumed large quantities of red meat was twice the number of individuals who did not consume red meat consumers in enterotype 1 but half the number in enterotypes 2 and type 3 (T1 vs. T2 + T3, $p=0.0081$ ) (Table 2. Additional file 2: Figure S1b). According to the results of the Bristol stool scale, feces shape

Table 2 Association between enterotypes and various other factors from the questionnaire

\begin{tabular}{|c|c|c|c|c|c|c|}
\hline & \multicolumn{3}{|l|}{ Enterotype } & \multicolumn{3}{|l|}{ Association } \\
\hline & $\begin{array}{l}\text { Type 1 } \\
(n=30)\end{array}$ & $\begin{array}{l}\text { Type 2 } \\
(n=36)\end{array}$ & $\begin{array}{l}\text { Type 3 } \\
(n=40)\end{array}$ & Contrast & $p$-value & $x^{2}$ \\
\hline Gender (global $p=0.047$ ) & & & & Type 1 vs Type 2 & 0.0336 & 4.52 \\
\hline male & $17(56.7 \%)$ & $10(27.2 \%)$ & $19(47.5 \%)$ & Type 1 vs Type 3 & 0.6046 & 0.27 \\
\hline \multirow[t]{2}{*}{ female } & $13(43.3 \%)$ & $26(72.2 \%)$ & $21(52.5 \%)$ & Type 2 vs Type 3 & 0.1258 & 2.34 \\
\hline & & & & Type 2 vs (Type $1+$ Type 3$)^{a}$ & 0.0200 & 5.41 \\
\hline Protein (global $p=0.015$ ) & & & & Type 1 vs Type 2 & 0.0290 & 4.77 \\
\hline non-red-meat & $8(32.0 \%)$ & $15(68.2 \%)$ & $18(66.7 \%)$ & Type 1 vs Type 3 & 0.0264 & 4.93 \\
\hline \multirow[t]{2}{*}{ red-meat } & 17 (68.0\%) & $7(31.8 \%)$ & $9(33.3 \%)$ & Type 2 vs Type 3 & 1 & 0.01 \\
\hline & & & & Type 1 vs. (Type $2+$ Type 3) ${ }^{a}$ & 0.0081 & 7.00 \\
\hline Shape (global $p=0.014$ ) & & & & Type 1 vs Type 2 & 0.6133 & 0.98 \\
\hline Hard & $10(41.7 \%)$ & $11(55.0 \%)$ & $3(11.1 \%)$ & Type 1 vs Type 3 & 0.0356 & 6.67 \\
\hline Mid & $8(33.3 \%)$ & $6(30.0 \%)$ & $11(40.7 \%)$ & Type 2 vs Type 3 & 0.0320 & 11.51 \\
\hline Watery & $6(25.0 \%)$ & $3(15.0 \%)$ & $13(48.1 \%)$ & (Type $1+$ Type 2 ) vs Type $3^{a}$ & 0.0038 & 11.15 \\
\hline Stool (global $p=0.064$ ) & & & & Type 1 vs Type 2 & 0.0384 & 6.52 \\
\hline D1+ & $17(58.6 \%)$ & $30(83.3 \%)$ & $30(81.1 \%)$ & Type 1 vs Type 3 & 0.0711 & 6.29 \\
\hline D05 & $11(37.9 \%)$ & $4(11.1 \%)$ & $5(13.5 \%)$ & Type 2 vs Type 3 & 0.9525 & 0.10 \\
\hline Constipation & $1(3.4 \%)$ & $2(5.6 \%)$ & $2(5.4 \%)$ & Type 1 vs (Type $2+$ Type 3$)^{a}$ & 0.0133 & 8.64 \\
\hline
\end{tabular}

${ }^{a}$ Combining two types based on no significant difference between groups and closed trend 
showed a significantly higher correlation with enterotype 3 than water content (T1 + T2 vs. T3, $p=0.0038$ ) (Table 2. Additional file 2: Figure S1c). Food digestion time (stool) in enterotype 1 was significantly higher than that in enterotypes 2 and 3 (T1 vs. T2 + T3, $p=0.0133$ ) (Table 2). In addition, a higher digestion time was strongly associated with a high red meat diet (D1+ vs. D05, $p<0.0001, \chi^{2}=$ 12.92) (Additional file 2: Figure S1d).

\section{Enterotype pathway enrichment analysis}

Enterotype 1 shows higher pathway activity than enterotype 2 or enterotype 3 in some KEGG pathways (ko00902, ko00909, ko05168, ko05416, ko05145, ko05210, ko04115, ko04610) (Additional file 3: Table S2). Two metabolic pathways are related to terpenoid biosynthesis; three pathways are related to infections such as virus and parasite; two pathways are associated with cancer and p53 DNA repair system; ko04610 is related to innate immune system.

\section{Classification of enterotypes}

To construct the decision tree model for classifying three enterotypes, 12 features were collected from 106 stool samples. These features include the Shannon index, F/A ratio, predominant genera and families, and enterotype-related phenotypes (gender, protein, shape, stool) (Additional file 4: Table S3). Figure 7 shows the result of the modeling. This decision tree interpreted five rules: 1) if the abundance of Prevotellaceae in a sample was greater than 0.26 , the sample was considered enterotype 3 ; 2) if the abundance of Prevotellaceae in a sample was lesser than 0.26 and the F/A ratio was greater than 0.2 , the sample was considered enterotype 1 ; 3) if the abundance of Prevotellaceae in a sample was lesser than 0.26, the abundance of Bacteroidaceae was greater than 0.33 , and the F/A ratio was lesser than 0.28 , the sample was considered enterotype $2 ; 4$ ) if the abundance of Prevotellaceae and Bacteroidaceae in a sample was lesser than 0.26 and 0.33 , respectively, the abundance of Enterobacteriaceae was greater than 0.10 , and the F/A ratio was lesser than than 0.28 , the sample was considered enterotype 3; and 5) if the abundance of Prevoteallaceae, Bacteroidaceae, and Enterobacteriaceae in a sample was lesser than $0.26,0.33$, and 0.10 , respectively, and the F/A ratio was lesser than 0.28 , the sample was considered enterotype 2. Rules 1-3 categorized $94.3 \%$ samples into three enterotypes. In total, 75 samples were used as independent testing sets to evaluate the performance of the decision tree model. Table 3 shows the performance of the training and testing sets, the accuracies of which were 97.2 and $84.0 \%$, respectively.

\section{Conclusions}

This is the first study to identify enterotypes in stools from Taiwanese adults. Our findings revealed a new subtype of an enterotype predominant by family Enterobacteriaceae. The identification of this new subtype may have been due to the ethnic group, dietary habits, and locations studied. A decision tree model of enterotypes was constructed, and the accuracies of the training and independent test used to fit the model were 97.2 and $84 \%$, respectively, which validated the model. Several associations between dietary habits and enterotype were identified in this study. Table 4 shows the predominant bacteria based on statistical hypothesis tests and several features. The microbial interaction network showed three bacteria (Escherichia, Salmonella, and Klebsiella), which belong to the same family (Enterobacteriaceae). In contrast to our findings, Enterobacteriaceae was found in a large number of patients with constipation from irritable bowel syndrome [14].

Enterotype-related phenotypes provide data for observing the gastrointestinal tract with the nature of continual

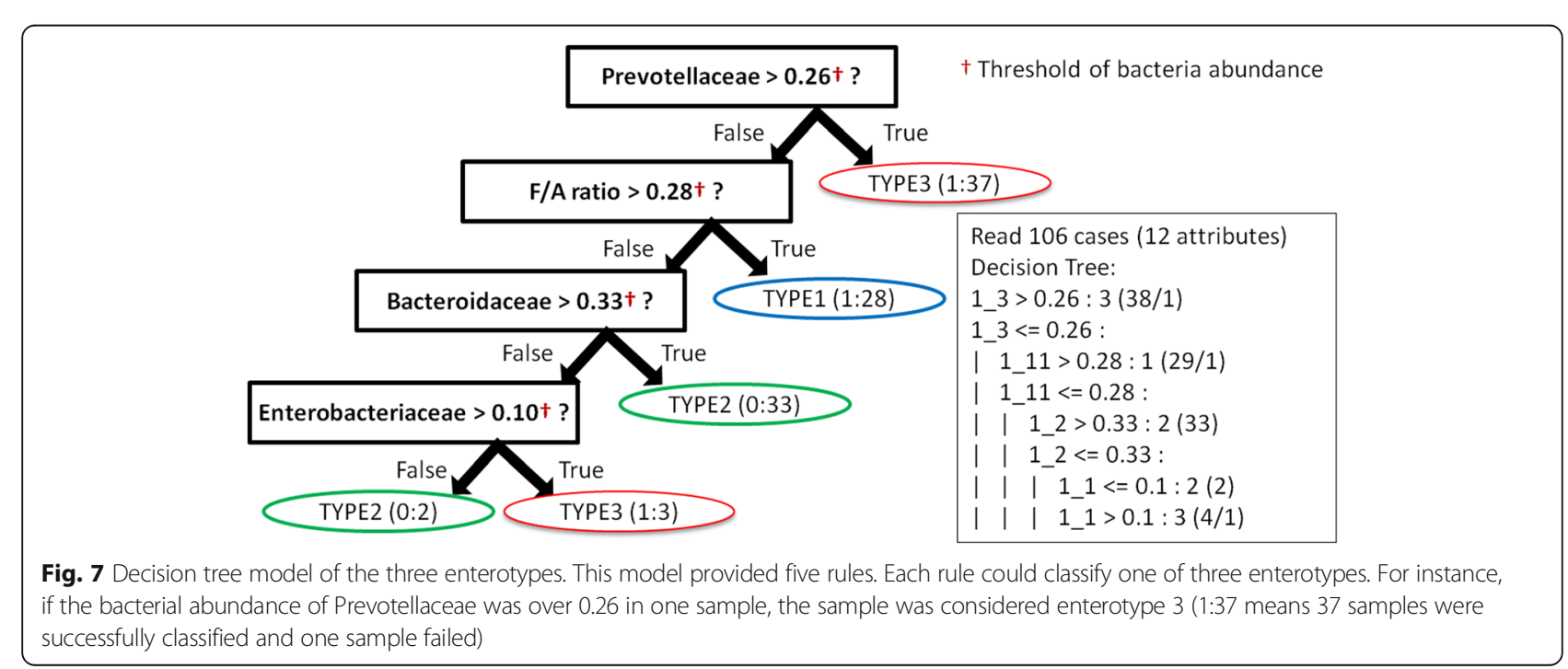


Table 3 Performance of classification model in training sets and independent testing sets

\begin{tabular}{llllll}
\hline & & T1 & T2 & T3 & Accuracy \\
\hline Sensitivity & Train & $93.3 \%(28 / 30)$ & $97.2 \%(35 / 36)$ & $100 \%(40 / 40)$ & $97.2 \%(103 / 106)$ \\
Group-specific ${ }^{a}$ specificity & Test & $93.8 \%(15 / 16)$ & $79.3 \%(23 / 29)$ & $83.3 \%(25 / 30)$ & $84.0 \%(63 / 75)$ \\
& Train & $98.7 \%(75 / 76)$ & $100 \%(70 / 70)$ & $97.0 \%(64 / 66)$ \\
Group-specific ${ }^{a}$ precision & Test & $88.1 \%(52 / 59)$ & $89.1 \%(41 / 46)$ & $100 \%(45 / 45)$ \\
& Train & $96.6 \%(28 / 29)$ & $100 \%(35 / 35)$ & $95.2 \%(40 / 42)$ \\
\hline
\end{tabular}

${ }^{a}$ Group-specific specificity, e.g., T1/non-T1

flux [7], e.g., nutrient substrate, water context, or transition status. With regard to stool frequency, the amount of Dialister and Akkermansia in "D05" was higher than that in "D1 +." According to previous studies, the amount of Dialister was higher in individuals with a high protein diet [15] and the amount of Akkermansia was higher in those with a fiber-free diet [16]. With regard to shape, the amounts of Parabacteroides and Prevotella in hard stool were higher than those in watery stool. Previous studies also showed that the amount of Prevotella was higher in ethnic groups that had a high fiber diet and lower in ethnic groups that adopted a Western diet [17]. With regard to protein type, the red meat group had abundance of Bifidobacterium and Akkermansia and the non-red meat group had abundance of Megamonas. Higher levels of lipid in the diet increased the amount of Bifidobacterium because it has the ability to digest lipids [18]. Enterotype 1 lacks of predominant bacteria such as Prevotella and Bacteroide, which may lead to a functional imbalance or a potential infectious risk via KEGG pathways (Additional file 3: Table S2).

Microflora, enterotype-related phenotypes, and shortchain fatty acids (SCFAs) were theoretically interwoven as a large association network [19]. Our study validates the connections among those factors (Additional file 5: Figure S2). SCFAs are byproducts of dietary fiber fermentation through microbiota, and they predominantly include acetic, propionic, and butyric acids. SCFAs can promote the growth of bacteria and can be absorbed by humans. Different types of SCFAs are sources of energy in different organs and are associated with intestinal diseases. Several factors control SCFA production in the gut, such as the amount and type of bacteria and the food retention time. The amounts of SCFAs affect $\mathrm{pH}$ of the intestine, for example, a higher concentration of SCFAs leads to lower $\mathrm{pH}$. The $\mathrm{pH}$ value is associated with the composition of the bacterial community. The complex interaction network in the gut includes the

Table 4 Significant genus lists categorized by enterotype-related metadata

\begin{tabular}{|c|c|c|c|c|}
\hline Features & Family & Genus & p. value & Mean separation \\
\hline \multirow[t]{8}{*}{ Enterotype } & Bacteroidaceae & Bacteroides & $<0.0001$ & $\mathrm{~T} 2>\mathrm{T} 1=\mathrm{T} 3$ \\
\hline & Prevotellaceae & Prevotella & $<0.0001$ & $\mathrm{~T} 3>\mathrm{T} 1>\mathrm{T} 2$ \\
\hline & Enterobacteriaceae & Escherichia & $<0.0001$ & $\mathrm{~T} 1>\mathrm{T} 2=\mathrm{T} 3$ \\
\hline & Enterobacteriaceae & Klebsiella & 0.0006 & $\mathrm{~T} 1>\mathrm{T} 2=\mathrm{T} 3$ \\
\hline & Enterobacteriaceae & Salmonella & 0.0060 & $\mathrm{~T} 1>\mathrm{T} 3$ \\
\hline & Bifidobacteriaceae & Bifidobacterrium & 0.0394 & $\mathrm{~T} 1>\mathrm{T} 3$ \\
\hline & Pseudomonadaceae & Pseudomonas & 0.0198 & $\mathrm{~T} 1>\mathrm{T} 2$ \\
\hline & Verrucomicrobiaceae & Akkermansia $^{\mathrm{b}}$ & 0.0017 & $\mathrm{~T} 1>\mathrm{T} 2=\mathrm{T} 3$ \\
\hline \multirow[t]{2}{*}{ Stool } & Veillonellaceae & Dialister & 0.0423 & $\mathrm{D} 1+<\mathrm{D} 05$ \\
\hline & Verrucomicrobiaceae & Akkermansia $^{b}$ & $0.0012^{\S}$ & $\mathrm{D} 1+<\mathrm{D} 05$ \\
\hline \multirow[t]{2}{*}{ Shape } & Porphyromonadaceae & Parabacteroides & 0.0106 & Hard $>$ Watery \\
\hline & Prevotellaceae & Prevotella & 0.0056 & Hard $>$ Watery \\
\hline \multirow[t]{3}{*}{ Protein } & Bifidobacteriaceae & Bifidobacterium & 0.0170 & Red $>$ non-red \\
\hline & Veillonellaceae & Megamonas & 0.0268 & Red $<$ non-red \\
\hline & Verrucomicrobiaceae & Akkermansia $^{\mathrm{b}}$ & 0.0042 & Red $>$ non-red \\
\hline
\end{tabular}

$\S p$ value was calculated by ANOVA (not significant in the Kolmogorov-Smirnov test)

${ }^{a}$ Genus within the same family

${ }^{\mathrm{b}}$ Genus associated with multiple metadata 
amount of SCFAs, $\mathrm{pH}$ value of the intestine, and composition of the bacterial community.

Our results provide a predictive model for further analysis and new insights into enterotypes. An individual may change his/her enterotype by making dietary changes because the characteristics of enterotypes depend on an individual's dietary habits. Although some researchers pointed that the gut microbiome should not category as 'Enterotypes or Faecotypes' since there is no clearly separation among clusters [20]. The classification may be blurred, yet the different features are still there. Thus, knowing one's enterotype may allow doctors to outline the best diet for patients and to prescribe the most effective drugs.

\section{Methods}

\section{Feces sample collection}

The 181 human feces samples used in this populationbased study were collected by Sigma-Transwab (Medical Wire). Feces were temporarily stored at $4{ }^{\circ} \mathrm{C}$ before DNA extraction. The exclusion criteria were age less than 10 years, a history of gastrointestinal tract surgery, and hospitalization or antibiotic treatment within the past 2 months. Of the resulting study cohort of 181 individuals, 106 provided complete information on the questionnaire and 75 omitted some information.

\section{DNA extraction}

In this case study, fresh feces were obtained from participants, and DNA was directly extracted from stool samples using the QIAamp DNA Stool Mini Kit (Qiagen). A swab sample was vigorously vortexed and incubated at room temperature for $1 \mathrm{~min}$. Then, the sample was transferred to a microcentrifuge tube containing $560 \mu \mathrm{l}$ Buffer ASL, vortexed, and incubated at $37^{\circ} \mathrm{C}$ for $30 \mathrm{~min}$. Following this, the suspension was incubated at $95{ }^{\circ} \mathrm{C}$ for $15 \mathrm{~min}$, vortexed, and centrifuged at $14,000 \mathrm{rpm}$ for $1 \mathrm{~min}$ into pellet stool particles. Extraction was performed following the protocol of the QIAamp DNA Stool Mini Kit. The DNA was eluted with $50 \mu \mathrm{l}$ Buffer AE and centrifuged at 14,000 rpm for $1 \mathrm{~min}$, after which the DNA extract was stored at $-20{ }^{\circ} \mathrm{C}$ until further analysis.

\section{Library construction and sequencing of the V4 region of the $16 \mathrm{~S}$ ribosomal DNA}

The PCR primers F515 (5'-GTGCCAGCMGCCGCGGTAA-3 $\left.{ }^{\prime}\right)$ and R806 (5'-GGACTACHVGGGTWTCTAAT-3') were designed to amplify the V4 region of the bacterial 16S ribosomal DNA as described previously [21]. PCR amplification was performed in a $50-\mu \mathrm{l}$ reaction volume containing $25 \mu \mathrm{l} 2 \mathrm{X}$ Taq Master Mix (Thermo Scientific), $0.2 \mu \mathrm{M}$ of forward and reverse primer, and 20 ng DNA template. The reaction process increased the initial temperature to $95{ }^{\circ} \mathrm{C}$ for $5 \mathrm{~min}$, followed by 30 cycles of $95{ }^{\circ} \mathrm{C}$ for $30 \mathrm{~s}, 54{ }^{\circ} \mathrm{C}$ for $1 \mathrm{~min}$, and $72{ }^{\circ} \mathrm{C}$ for $1 \mathrm{~min}$ as well as a final extension of $72{ }^{\circ} \mathrm{C}$ for $5 \mathrm{~min}$. Next, amplified products were checked by $2 \%$ agarose gel electrophoresis and ethidium bromide staining. Amplicons were purified using the AMPure XP beads (Agencourt) and quantified using the Qubit dsDNA HS Assay Kit (Thermo Fisher Scientific), all according to the respective manufacturers' instructions. For V4 library preparation, Illumina adapters were attached to the amplicons using the Illumina TruSeq DNA Sample Preparation v2 Kit. Purified libraries were processed for cluster generation and sequencing using the MiSeq system.

\section{Filtering $16 \mathrm{~S}$ rDNA sequencing data for quality}

Sequencing reads from different samples were identified and separated according to specific barcodes at the 5' end of the sequence (two mismatches allowed). The FASTX-Toolkit was employed to process the raw read data files. There were three steps used for sequence quality processing: (i) The command was "fastq_quality_filter -Q33-q 20-p 70." “-q 20" meant that he minimum quality score to be maintained is 20 . "-p 70 " meant that the minimum percent of bases must have "-q" quality over or equal to $70 \%$. (ii) The command was "fastq_quality_trimmer - $\mathrm{t} 20-1100-\mathrm{Q} 33$. ." “-t 20" meant that bases with lower quality $(<20)$ would be trimmed (checking from the end of the sequence). "-1 100 " meant that the minimum acceptable length of sequence was 100 after trimming the sequence. (iii) Sequences were retained if both forward and reverse sequencing reads passed the first and second steps.

\section{Taxonomy assignment for bacteria 16S rDNA sequence}

To generate taxonomy assignment, the collection of $16 \mathrm{~S}$ rDNA sequences was retrieved from the SILVA ribosomal RNA sequence database (release 115) [22]. These sequences were extracted using V4 forward and reverse primers. Then, UCLUST was used to create representative sequence clusters over or equal to $97 \%$ similarity [23]. Bowtie2 was used to align sequencing reads against the clusters of the V4 sequence. A 97\% similarity standard was applied to the V4 sequence clusters.

\section{Bacterial community analysis}

After taxonomy assignment, an OTU table was generated. To normalize the sample size of all samples, a rarefaction process was applied to the OTU table. There are three steps in deciphering the enterotype of stool samples [24]. The first step is to calculate the distance matrix of $\beta$-diversity. R package "vegan" [25] and Python software "Pycogent" [26] were employed to calculate nine types of matrices, namely Alternative Gower, Bray 
Cutris, Jaccard, Kulczynski, Chebyshev, Pearson, Horn, Euclidean, and Weighted UniFrac. The second step is to use these matrices as the input data for three cluster algorithms: HC, k-means clustering, and PAM methods. For PAM, there were two types of inputs: one was the distance matrix of $\beta$-diversity and the other was the point information of $\mathrm{XY}$ axes that were transformed from the distance matrix. $\mathrm{R}$ package was also used to perform the clustering process. The third step is to evaluate the quality of clustering results. Silhouette score was calculated by R package "clusterSim" [27]. A higher score represents better quality of clustering results. To explore the association between bacterial community and factors related to individuals, which were extracted from the questionnaires, weighted $\alpha$-diversity (Shannon index), chi-square test, and analysis of variance (ANOVA) were performed with $\mathrm{R}$ package. There were three criteria for identifying significant bacteria in the groups: the first was relative abundance $>1 \%$ in at least one group, the second was fold change in relative abundance between two groups $\geq \log _{2}(3)$ or $\leq \log _{2}(1 / 3)$. The third was $p$ value $\leq 0.05$. In order to construct a predictive model for classifying the three enterotypes of the stool samples, 181 stool samples were separated into two sets: the training set contained 106 samples, which were from individuals who provided complete information on the questionnaire, and the independent testing set contained 75 samples, which were from those who did not provide complete information. The decision tree, which was a rule-based machine learning method, was used to construct the predictive model for the three enterotypes. C4.5, which is a well-built decision tree package, was employed to perform this modeling process [28]. Tax4Fun was adopted to the pathway enrichment analysis with ANOVA [29].

\section{Additional files}

Additional file 1: Table S1. Statistics showing analysis results of 181 stool samples. (XLSX $16 \mathrm{~kb}$ )

Additional file 2: Figure S1. Association between enterotypes and several other factors such as Sex, Protein, Shape and Stool. (TIF $11718 \mathrm{~kb}$ )

Additional file 3: Table S2 Pathway enrichment analysis. (PDF 235 kb)

Additional file 4: Table S3. List of selected twelve features. (XLSX $12 \mathrm{~kb}$ )

Additional file 5: Figure S2. Theoretical network of microflora, substrates of microbes, and transit time in human gut microsystem. (TIF $213 \mathrm{~kb}$ )

\section{Acknowledgments}

We would like to thank the Come True Biomedical Inc. (CTB) for sharing their pearls of wisdom with us during the course of this research.

\section{Declarations}

This article has been published as part of BMC Genomics Volume 18 Supplement 1, 2016: Proceedings of the 27th International Conference on Genome Informatics: genomics. The full contents of the supplement are available online at http:// bmcgenomics.biomedcentral.com/articles/supplements/volume-18-supplement-1.

\section{Funding}

This project was funded by the Ministry of Science and Technology of the Republic of China [MOST 103-2628-B-009-001-MY3, MOST 104-2627-M-009008-, MOST 104-2319-B-400-002, MOST 104-2911-I-009-509, MOST 105-2633B-009-003, MOST 104-2314-B-195-014] and by the Ministry of Health and Welfare of the Republic of China [MOHW105-TDU-B-212-134002]. We also thank Veterans General Hospitals and University System of Taiwan (VGHUST) Joint Research Program [VGHUST 105-G1-4-2]. This paper is particularly supported by "Aiming for the Top University Program" of the National Chiao Tung University and Ministry of Education, Taiwan, R.O.C. The source of funding for the publication of the study is MOST 103-2628-B-009-001-MY3.

\section{Availability of data and materials}

The datasets supporting the results of this article are included within the article and its Additional files 1, 2, 3, 4 and 5.

\section{Authors' contributions}

$\mathrm{HDH}, \mathrm{CL}$, and HCT designed the study. HCT, SLW, CTW, KYL, and CHC lead the clinical study and sample collection. WCW, CMC, JYC, and HMW processed the samples and generated the sequence data. $\mathrm{HDH}, \mathrm{CL}$, WCW, and HMC analyzed and interpreted the data. HDH, WCW, THC, and HMC wrote the manuscript. All authors read and approved the final manuscript.

\section{Authors' information}

Not applicable.

\section{Competing interests}

The authors declare that they have no competing interests.

\section{Consent for publication}

Not applicable.

\section{Ethics approval and consent to participate}

Participants were recruited at Tseng Han-Chi General Hospital (Nantou, Taiwan). They volunteered to participate in the study. Before interviewing, the participants were informed or reminded of the purpose of the study and that they may withdraw at any time. Informed consent was obtained from all participants prior to enrolment in the study.

\section{Author details}

${ }^{1}$ Institute of Bioinformatics and Systems Biology, National Chiao Tung University, HsinChu, Taiwan. ${ }^{2}$ Tseng Han-Chi's General Hospital, Nantou, Taiwan. ${ }^{3}$ Health GeneTech Corporation, Taoyuan, Taiwan. ${ }^{4}$ Department of Biological Science and Technology, National Chiao Tung University, HsinChu, Taiwan. ${ }^{5}$ Department of Obstetrics and Gynecology, Hsinchu Mackay Memorial Hospital, Hsinchu, Taiwan. ${ }^{6}$ Mackay Medicine, Nursing and Management College, Taipei, Taiwan. ${ }^{7}$ Department of Medicine, Mackay Medical College, New Taipei City, Taiwan. ${ }^{8}$ Graduate Institute of Biomedical Informatics, Taipei Medical University, Taipei, Taiwan. ${ }^{9}$ School of Pharmacy, College of Pharmacy, Taipei Medical University, Taipei, Taiwan. ${ }^{10}$ Department of Biomedical Science and Environmental Biology, Kaohsiung Medical University, Kaohsiung, Taiwan.

Published: 25 January 2017

\section{References}

1. Aziz Q, Dore J, Emmanuel A, Guarner F, Quigley EM. Gut microbiota and gastrointestinal health: current concepts and future directions. Neurogastroenterol Motil. 2013;25(1):4-15.

2. Franzosa EA, Morgan XC, Segata N, Waldron L, Reyes J, Earl AM, Giannoukos G, Boylan MR, Ciulla D, Gevers D, et al. Relating the metatranscriptome and metagenome of the human gut. Proc Natl Acad Sci U S A. 2014;111(22): E2329-38.

3. Korecka A, Arulampalam V. The gut microbiome: scourge, sentinel or spectator? J Oral Microbiol. 2012;4:9367.

4. Curtis $\mathrm{H}$, et al. Structure, function and diversity of the healthy human microbiome. Nature. 2012;486(7402):207-14.

5. Koren O, Knights D, Gonzalez A, Waldron L, Segata N, Knight R, Huttenhower C, Ley RE. A guide to enterotypes across the human body: meta-analysis of microbial community structures in human microbiome datasets. PLoS Comput Biol. 2013;9(1):e1002863. 
6. Arumugam M, Raes J, Pelletier E, Le Paslier D, Yamada T, Mende DR, Fernandes GR, Tap J, Bruls T, Batto JM, et al. Enterotypes of the human gut microbiome. Nature. 2011;473(7346):174-80.

7. Zhou Y, Mihindukulasuriya KA, Gao H, La Rosa PS, Wylie KM, Martin JC, Kota K, Shannon WD, Mitreva M, Sodergren E, et al. Exploration of bacterial community classes in major human habitats. Genome Biol. 2014;15(5):R66.

8. Wu GD, Chen J, Hoffmann C, Bittinger K, Chen YY, Keilbaugh SA, Bewtra M, Knights D, Walters WA, Knight R, et al. Linking long-term dietary patterns with gut microbial enterotypes. Science. 2011;334(6052):105-8.

9. De Filippo C, Cavalieri D, Di Paola M, Ramazzotti M, Poullet JB, Massart S, Collini S, Pieraccini G, Lionetti P. Impact of diet in shaping gut microbiota revealed by a comparative study in children from Europe and rural Africa. Proc Natl Acad Sci U S A. 2010;107(33):14691-6.

10. Zupancic ML, Cantarel BL, Liu Z, Drabek EF, Ryan KA, Cirimotich S, Jones C, Knight $R$, Walters WA, Knights $D$, et al. Analysis of the gut microbiota in the old order Amish and its relation to the metabolic syndrome. PLOS ONE. 2012;7(8):e43052.

11. Huse SM, Ye Y, Zhou Y, Fodor AA. A core human microbiome as viewed through 16S rRNA sequence clusters. PLoS ONE. 2012;7(6):e34242.

12. Mondot S, de Wouters T, Dore J, Lepage P. The human gut microbiome and its dysfunctions. Dig Dis. 2013;31(3-4):278-85.

13. Lewis $\mathrm{SJ}$, Heaton KW. Stool form scale as a useful guide to intestinal transit time. Scand J Gastroenterol. 1997;32(9):920-4.

14. Chassard C, Dapoigny M, Scott KP, Crouzet L, Del'homme C, Marquet P, Martin JC, Pickering G, Ardid D, Eschalier A, et al. Functional dysbiosis within the gut microbiota of patients with constipated-irritable bowel syndrome. Aliment Pharmacol Ther. 2012;35(7):828-38.

15. Hooda S, Vester Boler BM, Kerr KR, Dowd SE, Swanson KS. The gut microbiome of kittens is affected by dietary protein:carbohydrate ratio and associated with blood metabolite and hormone concentrations. Br J Nutr. 2013;109(9):1637-46.

16. Jakobsdottir G, Xu J, Molin G, Ahrne S, Nyman M. High-fat diet reduces the formation of butyrate, but increases succinate, inflammation, liver fat and cholesterol in rats, while dietary fibre counteracts these effects. PLoS ONE. 2013:8(11):e80476

17. Pop M, Walker AW, Paulson J, Lindsay B, Antonio M, Hossain MA, Oundo J, Tamboura B, Mai V, Astrovskaya I, et al. Diarrhea in young children from low-income countries leads to large-scale alterations in intestinal microbiota composition. Genome Biol. 2014;15(6):R76.

18. Bordoni A, Amaretti A, Leonardi A, Boschetti E, Danesi F, Matteuzzi D, Roncaglia L, Raimondi S, Rossi M. Cholesterol-lowering probiotics: in vitro selection and in vivo testing of bifidobacteria. Appl Microbiol Biotechnol. 2013;97(18):8273-81.

19. Hijova E, Chmelarova A. Short chain fatty acids and colonic health. Bratisl Lek Listy. 2007;108(8):354-8.

20. Jeffery IB, Claesson MJ, O'Toole PW, Shanahan F. Categorization of the gut microbiota: enterotypes or gradients? Nat Rev Microbiol. 2012;10(9):591-2.

21. Weng SL, Chiu CM, Lin FM, Huang WC, Liang C, Yang T, Yang TL, Liu CY, Wu WY, Chang YA, et al. Bacterial communities in semen from men of infertile couples: metagenomic sequencing reveals relationships of seminal microbiota to semen quality. PLoS ONE. 2014:9(10):e110152.

22. Pruesse E, Quast C, Knittel K, Fuchs BM, Ludwig W, Peplies J, Glockner FO. SILVA: a comprehensive online resource for quality checked and aligned ribosomal RNA sequence data compatible with ARB. Nucleic Acids Res. 2007:35(21):7188-96.

23. Edgar RC. Search and clustering orders of magnitude faster than BLAST. Bioinformatics. 2010;26(19):2460-1.

24. Wang J, Linnenbrink M, Kunzel S, Fernandes R, Nadeau MJ, Rosenstiel P, Baines JF. Dietary history contributes to enterotype-like clustering and functional metagenomic content in the intestinal microbiome of wild mice. Proc Natl Acad Sci U S A. 2014;111(26):E2703-10.

25. Dixon P. VEGAN, a package of $R$ functions for community ecology. J Veg Sci. 2003;14:927-30.

26. Knight R, Maxwell P, Birmingham A, Carnes J, Caporaso JG, Easton BC, Eaton M, Hamady M, Lindsay H, Liu Z, et al. PyCogent: a toolkit for making sense from sequence. Genome Biol. 2007;8(8):R171.

27. Walesiak M. Cluster analysis with ClusterSim computer program and $\mathrm{R}$ environment. Acta Universitatis Lodziensis: Folia Oeconomica. 2008;216:303-11.

28. Quinlan JR. C4.5: programs for machine learning. San Mateo: Morgan Kaufmann Publishers; 1993.

29. Asshauer KP, Wemheuer B, Daniel R, Meinicke P. Tax4Fun: predicting functional profiles from metagenomic 16S rRNA data. Bioinformatics. 2015;31(17):2882-4.

\section{Submit your next manuscript to BioMed Central and we will help you at every step:}

- We accept pre-submission inquiries

- Our selector tool helps you to find the most relevant journal

- We provide round the clock customer support

- Convenient online submission

- Thorough peer review

- Inclusion in PubMed and all major indexing services

- Maximum visibility for your research

Submit your manuscript at www.biomedcentral.com/submit 